\title{
Barycenters in the Wasserstein space
}

\author{
Martial Agueh *, Guillaume Carlier ${ }^{\dagger}$
}

December 10, 2010

\begin{abstract}
In this paper, we introduce a notion of barycenter in the Wasserstein space which generalizes McCann's interpolation to the case of more than two measures. We provide existence, uniqueness, characterizations and regularity of the barycenter, and relate it to the multimarginal optimal transport problem considered by Gangbo and Święch in [8]. We also consider some examples and in particular rigorously solve the gaussian case. We finally discuss convexity of functionals in the Wasserstein space.
\end{abstract}

Keywords: Optimal transport, Wasserstein space, convexity, duality.

AMS Subject Classifications:. 49J40, 49K21, 49K30.

\section{Introduction}

In this paper, we consider a nonlinear interpolation between several probability measures on $\mathbb{R}^{d}$. By analogy with the Euclidean case where the barycenter of points $\left(x_{1}, \ldots, x_{p}\right)$ with barycentric coordinates $\left(\lambda_{1}, . ., \lambda_{p}\right)$ is obtained as the minimizer of $x \mapsto \sum_{i=1}^{p} \lambda_{i}\left|x-x_{i}\right|^{2}$, we propose the same procedure in the Wasserstein space by simply replacing the squared euclidean distance with the squared 2-Wasserstein distance. The notion of barycenter as a minimizer of an averaged squared distance is not new and has already been investigated in depth by Sturm [14] in the framework of nonpositively curved metric spaces. It turns out however that the Wasserstein space is not nonpositively

\footnotetext{
* Department of Mathematics and statistics University of Victoria, Victoria, BC, PO Box 3060 STN CSC Victoria, BC, V8W 3R4, CANADA, agueh@math.uvic.ca.

${ }^{\dagger}$ CEREMADE, UMR CNRS 7534, Université Paris IX Dauphine, Pl. de Lattre de Tassigny, 75775 Paris Cedex 16, FRANCE carlier@ceremade.dauphine.fr.
} 
curved as illustrated by example 7.3.3 in the book of Ambrosio, Gigli and Savare [1] and that much less is known on existence, uniqueness and properties of barycenters outside of the nonpositively curved case (see however the recent article of Ohta [12] for the case of Alexandrov spaces of curvature bounded from below). The properties of barycenters in the Wasserstein space (uniqueness issues in particular) therefore deserve in our opinion to be studied in their own right.

In the case of two probability measures, such an interpolation is already known as the McCann's interpolation [11] that led to the concept of displacement convexity that has proved to be a very powerful tool in the theory of gradient flows in the Wasserstein space as explained in [1]. Our interest in interpolating between more than two probability measures by minimizing an averaged sum of squared Wasserstein distances originated from the recent papers in mathematical economics by Carlier and Ekeland [4], and Chiappori, McCann and Nesheim [5] who studied matching and hedonic pricing problems by means of optimal transport techniques. Interestingly, such problems also arise in a very different applied setting in image processing for which we refer to the recent paper of Bernot, Delon, Peyré and Rabin [2].

We will therefore study the following minimization problem

$$
\inf _{\nu} \sum_{i=1}^{p} \lambda_{i} W_{2}^{2}\left(\nu_{i}, \nu\right)
$$

where the $\nu_{i}$ 's are probability measures with finite second moments, the $\lambda_{i}$ 's are positive weights summing to 1 , and $W_{2}^{2}$ denotes the squared 2-Wasserstein distance. We will establish existence and uniqueness (provided one of the $\nu_{i}$ 's vanishes on small sets) of the solution to (1.1), and we will naturally refer to it as the barycenter of the measures $\nu_{i}$ 's with weights $\lambda_{i}$ 's. We will also provide characterization and regularity results, and finally discuss some examples.

In section 2, we introduce a dual problem to (1.1) from which optimality conditions and uniqueness of the barycenter are derived in section 3. Section 4 relates the barycenter problem to the quadratic multi-marginal optimal transport problem considered by Gangbo and Święch in [8]. In Section 5, we establish an $L^{\infty}$ regularity results for the barycenter. Section 6 is devoted to some examples. Finally, section 7 discusses various convexity properties of functionals of measures. 


\section{The primal problem and its dual}

The space of continuous functions with at most quadratic growth,

$$
Y:=\left(1+|\cdot|^{2}\right) C_{b}\left(\mathbb{R}^{d}\right)=\left\{f \in C\left(\mathbb{R}^{d}\right): \frac{f}{1+|\cdot|^{2}} \text { is bounded }\right\},
$$

will be equipped with the norm

$$
\|f\|_{Y}:=\sup _{x \in \mathbb{R}^{d}} \frac{|f(x)|}{1+|x|^{2}}
$$

Let $X$ be the closed subspace of $Y$ given by

$$
X:=\left(1+|\cdot|^{2}\right) C_{0}\left(\mathbb{R}^{d}\right)=\left\{f \in C\left(\mathbb{R}^{d}\right): \lim _{|x| \rightarrow \infty} \frac{f(x)}{1+|x|^{2}}=0\right\} .
$$

We denote by $\mathcal{M}\left(\mathbb{R}^{d}\right)$ the space of bounded Radon measures on $\mathbb{R}^{d}$, as usual identified with the dual of $C_{0}\left(\mathbb{R}^{d}\right)$ (space of continuous functions that vanish at infinity) and by $\mathcal{M}_{+}^{1}\left(\mathbb{R}^{d}\right)$ the set of Radon probability measures on $\mathbb{R}^{d}$. We shall naturally identify the dual of $X$ with

$$
X^{\prime}=\left\{\mu \in \mathcal{M}\left(\mathbb{R}^{d}\right):\left(1+|x|^{2}\right) \mu \in \mathcal{M}\left(\mathbb{R}^{d}\right)\right\} .
$$

Given two probability measures with finite second moments $\mu$ and $\nu$ (i.e. $\mu$ and $\nu$ in $X^{\prime} \cap \mathcal{M}_{+}^{1}\left(\mathbb{R}^{d}\right)$ ), the 2-Wasserstein distance between $\mu$ and $\nu$, $W_{2}(\mu, \nu)$, is defined as the value of the following Monge-Kantorovich optimal transportation problem:

$$
W_{2}^{2}(\mu, \nu):=\inf \left\{\int_{\mathbb{R}^{d} \times \mathbb{R}^{d}}|x-y|^{2} d \gamma(x, y), \gamma \in \Pi(\mu, \nu)\right\}
$$

where $\Pi(\mu, \nu)$ denotes the set of transport plans between $\mu$ and $\nu$ i.e. the set of probability measures on $\mathbb{R}^{d} \times \mathbb{R}^{d}$ having $\mu$ and $\nu$ as marginals.

Given an integer $p \geq 2, p$ probability measures $\nu_{1}, \ldots, \nu_{p}$ in $X^{\prime} \cap \mathcal{M}_{+}^{1}\left(\mathbb{R}^{d}\right)$ and $p$ real numbers $\lambda_{1}, \ldots \lambda_{p}$ such that $\lambda_{i}>0$ and $\sum_{i=1}^{p} \lambda_{i}=1$, we are interested in the following problem:

$$
(\mathcal{P}) \inf _{\nu \in X^{\prime} \cap \mathcal{M}_{+}^{1}\left(\mathbb{R}^{d}\right)} J(\nu)=\sum_{i=1}^{p} \frac{\lambda_{i}}{2} W_{2}^{2}\left(\nu_{i}, \nu\right) .
$$

In analogy with the Euclidean case, a solution of the previous problem will be called the barycenter of the probabilities $\nu_{i}$ with weights $\lambda_{i}$. 
To study existence and uniqueness of the barycenter, we introduce the dual (this terminology will be justified later on) of $(\mathcal{P})$,

$$
\left(\mathcal{P}_{0}^{*}\right) \sup \left\{F\left(f_{1}, \ldots, f_{p}\right)=\sum_{i=1}^{p} \int_{\mathbb{R}^{d}} S_{\lambda_{i}} f_{i} d \nu_{i}: \sum_{i=1}^{p} f_{i}=0, f_{i} \in X\right\}
$$

where

$$
S_{\lambda} f(x):=\inf _{y \in \mathbb{R}^{d}}\left\{\frac{\lambda}{2}|x-y|^{2}-f(y)\right\}, \forall x \in \mathbb{R}^{d}, f \in X, \lambda>0 .
$$

For $i \in\{1, \cdots, p\}$, we define

$$
H_{i}(f):=-\int_{\mathbb{R}^{d}} S_{\lambda_{i}} f(x) d \nu_{i}(x) .
$$

Note that $S_{\lambda} f$ defined as above may take the value $-\infty$. It is easy to check that $H_{i}$ is convex and l.s.c. on $Y$. By definition, the Legendre-Fenchel transform of $H_{i}$ is

$$
\begin{aligned}
H_{i}^{*}(\nu) & :=\sup _{f \in X}\left\{\int_{\mathbb{R}^{d}} f d \nu-H_{i}(f)\right\} \\
& =\sup _{f \in X}\left\{\int_{\mathbb{R}^{d}} f d \nu+\int_{\mathbb{R}^{d}} S_{\lambda_{i}} f d \nu_{i}\right\}, \forall \nu \in X^{\prime} .
\end{aligned}
$$

Since the supremum in $\left(\mathcal{P}_{0}^{*}\right)$ may not be attained, we consider its "relaxed" problem

$$
\left(\mathcal{P}^{*}\right) \sup \left\{F\left(f_{1}, \ldots, f_{p}\right): \sum_{i=1}^{p} f_{i}=0, f_{i} \in Y\right\} .
$$

Now, let $\nu \in X^{\prime} \cap \mathcal{M}_{+}^{1}\left(\mathbb{R}^{d}\right), \gamma_{i} \in \Pi\left(\nu_{i}, \nu\right)$ and $\left(f_{1}, \ldots, f_{p}\right) \in X^{p}$ which sum to 0 . Integrating the inequality

$$
S_{\lambda_{i}} f_{i}\left(x_{i}\right)+f_{i}(y) \leq \frac{\lambda_{i}}{2}\left|x_{i}-y\right|^{2}
$$

with respect to $\gamma_{i}$ and summing over $i$, we have after using that $\sum_{i=1}^{p} f_{i}=0$,

$$
\sum_{i=1}^{p} \int_{\mathbb{R}^{d}} S_{\lambda_{i}} f_{i} d \nu_{i} \leq \sum_{i=1}^{p} \frac{\lambda_{i}}{2} \int_{\mathbb{R}^{d} \times \mathbb{R}^{d}}\left|x_{i}-y\right|^{2} d \gamma_{i}\left(x_{i}, y\right) .
$$

We then deduce that

$$
\inf (\mathcal{P}) \geq \sup \left(\mathcal{P}^{*}\right) \geq \sup \left(\mathcal{P}_{0}^{*}\right)
$$

To prove the converse inequality, we first establish the following intermediate result: 
Lemma 2.1. For every $\nu \in X^{\prime}$, one has

$$
H_{i}^{*}(\nu)= \begin{cases}\frac{\lambda_{i}}{2} W_{2}^{2}\left(\nu_{i}, \nu\right) & \text { if } \nu \in X^{\prime} \cap \mathcal{M}_{+}^{1}\left(\mathbb{R}^{d}\right) \\ +\infty & \text { otherwise. }\end{cases}
$$

Proof. We first prove that $H_{i}^{*}(\nu)=+\infty$ whenever $\nu \in X^{\prime} \backslash \mathcal{M}_{+}^{1}\left(\mathbb{R}^{d}\right)$. Indeed, if $\nu$ is not nonnegative, there exists $f \in X, f \leq 0$ such that $\int_{\mathbb{R}^{d}} f d \nu>0$. Then $S_{\lambda_{i}}(t f) \geq 0$ for every $t \geq 0$, and we have

$$
H_{i}^{*}(\nu) \geq \sup _{t \geq 0} t \int_{\mathbb{R}^{d}} f d \nu=+\infty
$$

But if $\nu \in X^{\prime}$ is nonnegative and $\nu\left(\mathbb{R}^{d}\right) \neq 1$, say $\nu\left(\mathbb{R}^{d}\right)<1$, using $f(x)=-t$ with $t \geq 0$ in $H_{i}^{*}(\nu)$, we have

$$
H_{i}^{*}(\nu) \geq \sup _{t \geq 0} t\left(-\int_{\mathbb{R}^{d}} d \nu+\int_{\mathbb{R}^{d}} d \nu_{i}\right)=\left(1-\nu\left(\mathbb{R}^{d}\right)\right) \sup _{t \geq 0} t=+\infty .
$$

We conclude that $H_{i}^{*}(\nu)=+\infty$ whenever $\nu \in X^{\prime} \backslash \mathcal{M}_{+}^{1}\left(\mathbb{R}^{d}\right)$.

Now let $\nu \in X^{\prime} \cap \mathcal{M}_{+}^{1}\left(\mathbb{R}^{d}\right)$. The well-known Kantorovich duality formula (see for instance [15]) reads as:

$$
\begin{aligned}
\frac{\lambda_{i}}{2} W_{2}^{2}\left(\nu, \nu_{i}\right) & =\sup \left\{\int_{\mathbb{R}^{d}} f d \nu+\int_{\mathbb{R}^{d}} g d \nu_{i}, f, g \in C_{b}, f(x)+g(y) \leq \frac{\lambda_{i}}{2}|x-y|^{2}\right\} \\
& =\sup _{f \in X}\left\{\int_{\mathbb{R}^{d}} f d \nu+\int_{\mathbb{R}^{d}} S_{\lambda_{i}} f d \nu_{i}\right\}=H_{i}^{*}(\nu) .
\end{aligned}
$$

This ends the proof.

We then easily deduce the following duality result:

\section{Proposition 2.2.}

$$
\inf (\mathcal{P})=\sup \left(\mathcal{P}_{0}^{*}\right)=\sup \left(\mathcal{P}^{*}\right)
$$

Proof. Thanks to lemma 2.1, we have on the one hand

$$
\inf (\mathcal{P})=\inf _{X^{\prime}} \sum_{i=1}^{p} H_{i}^{*}=-\left(\sum_{i=1}^{p} H_{i}^{*}\right)^{*}
$$

Defining $H$ as the inf-convolution of $H_{1}, \ldots, H_{p}$,

$$
H(f)=\left(\prod_{i=1}^{p} H_{i}\right)(f):=\inf \left\{\sum_{i=1}^{p} H_{i}\left(f_{i}\right): f_{i} \in X, \sum_{i=1}^{p} f_{i}=f\right\}, \forall f \in X
$$


we have on the other hand

$$
\sup \left(\mathcal{P}_{0}^{*}\right)=-H(0)
$$

It is a well-known fact of convex analysis (which can be checked by a straightforward computation) that

$$
H^{*}=\left(\prod_{i=1}^{p} H_{i}\right)^{*}=\sum_{i=1}^{p} H_{i}^{*}
$$

Then

$$
\inf (\mathcal{P})=-H^{* *}(0) \geq-H(0)=\sup \left(\mathcal{P}_{0}^{*}\right) .
$$

Hence, using (2.5), the desired result amounts to prove that $H(0)=H^{* *}(0)$. And since $H$ is convex, it is enough (see [7], propositions 3.3 and 4.1) to show that $H$ has a continuous affine minorant and is l.s.c. at 0 for the norm topology of $X$ (i.e. the topology induced by that of $Y$ ). To prove that, we rewrite $H_{i}$ as

$$
H_{i}\left(f_{i}\right)=\int_{\mathbb{R}^{d}} \sup _{y \in \mathbb{R}^{d}}\left\{f_{i}(y)-\frac{\lambda_{i}}{2}|x-y|^{2}\right\} d \nu_{i}(x),
$$

which yields in particular

$$
H_{i}\left(f_{i}\right) \geq f_{i}(0)-\frac{\lambda_{i}}{2} \int_{\mathbb{R}^{d}}|x|^{2} d \nu_{i}(x)
$$

so that

$$
H(f) \geq f(0)-\sum_{i=1}^{p} \frac{\lambda_{i}}{2} \int_{\mathbb{R}^{d}}|x|^{2} d \nu_{i}(x)>-\infty, \forall f \in X
$$

which gives a continuous affine minorant for $H$. Now, let $f \in Y$ be such that $4\|f\|_{Y} \leq p \min \left\{\lambda_{1}, \ldots, \lambda_{p}\right\}$. Choosing $f_{i}=f / p$ in $H(f)$, we have

$$
\begin{aligned}
H(f) & \leq \sum_{i=1}^{p} H_{i}\left(\frac{f}{p}\right) \leq \sum_{i=1}^{p} \int_{\mathbb{R}^{d}} \sup _{y \in \mathbb{R}^{d}}\left\{\frac{\lambda_{i}}{4}\left(1+|y|^{2}\right)-\frac{\lambda_{i}}{2}|x-y|^{2}\right\} d \nu_{i}(x) \\
& =\sum_{i=1}^{p} \int_{\mathbb{R}^{d}}\left(\frac{\lambda_{i}}{4}+\frac{\lambda_{i}}{2}|x|^{2}\right) d \nu_{i}(x) \\
& =\frac{1}{4}+\sum_{i=1}^{p} \frac{\lambda_{i}}{2} \int_{\mathbb{R}^{d}}|x|^{2} d \nu_{i}(x) .
\end{aligned}
$$

Note that we have used in the above computations that $f(y) \leq \frac{p \lambda_{i}}{4}\left(1+|y|^{2}\right)$ and $\sum_{i=1}^{p} \lambda_{i}=1$. We thus proved that the convex function $H$ never takes 
the value $-\infty$ and is bounded from above in a neighbourhood of 0 in $Y$. By a standard convex analysis result (see [7], proposition 2.5), it is therefore continuous at 0 . Hence, $H(0)=H^{* *}(0)$ and the result follows.

The next proposition gives existence of an optimizer to the primal problem $(\mathcal{P})$ and its dual $\left(\mathcal{P}^{*}\right)$.

Proposition 2.3. Both problems $(\mathcal{P})$ and $\left(\mathcal{P}^{*}\right)$ have solutions.

Proof. Let $\nu^{n}$ be a minimizing sequence of $(\mathcal{P})$. It is easy (for instance, by using (2.6)) to check that $\int_{\mathbb{R}^{d}}|x|^{2} d \nu^{n}(x)$ is bounded; hence $\nu^{n}$ is tight. It then follows from Prokhorov's Theorem (see [6]) that a (non relabeled) subsequence converges narrowly to some $\nu \in \mathcal{M}_{+}^{1}\left(\mathbb{R}^{d}\right)$, and it is easy to check that $\nu \in X^{\prime}$. Using (2.6), we then immediately get $J(\nu) \leq \liminf J\left(\nu^{n}\right)=$ $\inf (\mathcal{P})$, which shows that $(\mathcal{P})$ has a minimizer.

Proving the existence of a solution to $\left(\mathcal{P}^{*}\right)$ requires some preliminaries. First we show that in $\left(\mathcal{P}^{*}\right)$, one can choose the $f_{i}$ such that $\left(S_{\lambda_{i}} \circ S_{\lambda_{i}}\right) f_{i}=f_{i}$ for all $i=1, \cdots, p-1$. Indeed, if $\left(f_{1}, \ldots, f_{p}\right)$ is admissible for $\left(\mathcal{P}^{*}\right)$, then setting $g_{i}:=S_{\lambda_{i}} f_{i}, h_{i}:=S_{\lambda_{i}} g_{i}$ for all $i$, it is easy to check that $f_{i} \leq h_{i}$, $h_{i} \in X$ and $S_{\lambda_{i}} f_{i}=S_{\lambda_{i}} h_{i}$. Now, define $\left(\tilde{f}_{1}, \ldots, \widetilde{f}_{p-1}\right):=\left(h_{1}, \ldots, h_{p-1}\right)$ and $\tilde{f}_{p}:=-\sum_{i=1}^{p-1} \widetilde{f}_{i}$. Since $f_{i} \leq \widetilde{f}_{i}$ for $i=1, \ldots, p-1$, then $\tilde{f}_{p} \leq-\sum_{i=1}^{p-1} f_{i}=f_{p}$ . For $i=1, \ldots, p-1$, we then have $S_{\lambda_{i}} \widetilde{f}_{i}=S_{\lambda_{i}} f_{i}$, and since $S_{\lambda_{p}}$ is orderreversing, then $S_{\lambda_{p}} \widetilde{f}_{p} \geq S_{\lambda_{p}} f_{p}$ which shows that $F\left(\widetilde{f}_{1}, \ldots, \widetilde{f}_{p}\right) \geq F\left(f_{1}, \ldots, f_{p}\right)$. This proves that in $\left(\mathcal{P}^{*}\right)$, one may assume that $f_{i}=S_{\lambda_{i}} g_{i}$ with $g_{i}=S_{\lambda_{i}} f_{i}$ for $i=1, \ldots, p-1$. The relation $f_{i}=S_{\lambda_{i}} g_{i}$ can equivalently be written as

$$
\frac{\lambda_{i}}{2}|y|^{2}-f_{i}(y)=\sup _{x \in \mathbb{R}^{d}}\left\{\lambda_{i} x \cdot y-\frac{\lambda_{i}}{2}|x|^{2}+g_{i}(x)\right\}
$$

which implies that $\frac{\lambda_{i}}{2}|.|^{2}-f_{i}$ is convex for $i=1, \ldots, p-1$. Finally, we remark that, since the functional $F$ and the constraint of $\left(\mathcal{P}^{*}\right)$ are invariant, when one adds constants that sum to 0 to the $f_{i}$ 's, there is no loss of generality in assuming $f_{i}(0)=0$ for all $i$.

Now let $f^{n}:=\left(f_{1}^{n}, . ., f_{p}^{n}\right)$ be a maximizing sequence for $\left(\mathcal{P}^{*}\right)$ which, as explained before, can be chosen such that $f^{n}(0)=0$ and $f_{i}^{n}=S_{\lambda_{i}} g_{i}^{n}$ for $i=1, \ldots, p-1$ with $g_{i}^{n}:=S_{\lambda_{i}} f_{i}^{n}$ for $i=1, \ldots, p$. In particular, $\frac{\lambda_{i}}{2}|\cdot|^{2}-f_{i}^{n}$ is convex for $i=1, \ldots, p-1$ and every $n$. Using the fact that $f_{i}^{n}(0)=0$ and $g_{i}^{n}:=S_{\lambda_{i}} f_{i}^{n}$ we then have

$$
g_{i}^{n}(x) \leq \frac{\lambda_{i}}{2}|x|^{2}
$$


Since $f^{n}$ is a maximizing sequence for $\left(\mathcal{P}^{*}\right)$, there exists a constant $C_{1}$ such that

$$
\sum_{i=1}^{p} \int_{\mathbb{R}^{d}} g_{i}^{n}(x) d \nu_{i}(x) \geq C_{1}, \forall n .
$$

Then (2.7) and (2.8) imply that for all $j=1, \cdots, p$,

$$
\begin{aligned}
\int_{\mathbb{R}^{d}} g_{j}^{n}(x) d \nu_{j}(x) & \geq C_{1}-\sum_{i \neq j} \int_{\mathbb{R}^{d}} g_{i}^{n}(x) d \nu_{i}(x) \\
& \geq C_{1}-\sum_{i \neq j} \frac{\lambda_{i}}{2} \int_{\mathbb{R}^{d}}|x|^{2} d \nu_{i}(x) .
\end{aligned}
$$

And since the $\nu_{i}$ have finite second moments, we deduce from (2.7) and (2.9) that the integrals $\int_{\mathbb{R}^{d}} g_{i}^{n}(x) d \nu_{i}(x)$ are bounded. Now integrating the inequality $f_{i}^{n}(y) \leq \lambda_{i}|x-y|^{2} / 2-g_{i}^{n}(x)$ with respect to $\nu_{i}(x)$ and using the bound on $\int_{\mathbb{R}^{d}} g_{i}^{n}(x) d \nu_{i}(x)$, we see that there exists a constant $C_{2}$ such that

$$
f_{i}^{n}(y) \leq C_{2}\left(1+|y|^{2}\right), \forall i, \forall n, \forall y \in \mathbb{R}^{d} .
$$

Applying the above inequality to $f_{p}^{n}$ and recalling that $f_{p}^{n}=-\sum_{i=1}^{p-1} f_{i}^{n}$, we also have

$$
\sum_{i=1}^{p-1} f_{i}^{n}(y) \geq-C_{2}\left(1+|y|^{2}\right), \forall n, \forall y \in \mathbb{R}^{d} .
$$

Then we deduce by using again (2.10) that

$$
f_{i}^{n}(y) \geq-C_{2}\left(1+|y|^{2}\right)-\sum_{j \neq i} f_{j}^{n}(y) \geq-p C_{2}\left(1+|y|^{2}\right), \forall i, \forall n, \forall y \in \mathbb{R}^{d} .
$$

The subsequent inequality combined with (2.10) show that $f_{i}^{n}$ is bounded in $Y$ for every $i$. Since $\frac{\lambda_{i}}{2}||^{2}-f_{i}^{n}$ is convex for every $n$ and $i=1, \ldots, p-1$, passing to a subsequence if necessary, we may assume that $f^{n}$ converges uniformly on compact subsets to some $f=\left(f_{1}, \ldots f_{p}\right)$ with each $f_{i} \in Y$ (indeed, if a sequence of convex functions is bounded on the ball $B_{2 r}$ of center 0 and radius $r$ then it is equi-Lipschitz on the ball $B_{r}$ and one can therefore invoke Ascoli-Arzelà's theorem together with a diagonal extraction argument). Finally, the fact that $f$ solves $\left(\mathcal{P}^{*}\right)$ follows from Fatou's Lemma, the positivity of $\frac{\lambda_{i}}{2}|\cdot|^{2}-g_{i}^{n}$ and the inequality

$$
\begin{aligned}
\underset{n}{\limsup } g_{i}^{n}(x) & \leq \inf _{y \in \mathbb{R}^{d}}\left\{\limsup _{n}\left(\frac{\lambda_{i}}{2}|x-y|^{2}-f_{i}^{n}(y)\right)\right\} \\
& =\inf _{y \in \mathbb{R}^{d}}\left\{\frac{\lambda_{i}}{2}|x-y|^{2}-f_{i}(y)\right\}=S_{\lambda_{i}} f_{i}(x) .
\end{aligned}
$$




\section{Characterization of barycenters}

With the results of the previous section at hand, namely,

$$
\min (\mathcal{P})=\max \left(\mathcal{P}^{*}\right)
$$

our aim now is to further exploit this duality to characterize the barycenters i.e. the solution of $(\mathcal{P})$.

Let $\left(f_{1}, \ldots, f_{p}\right)$ be a solution of $\left(\mathcal{P}^{*}\right)$. It follows from the previous duality relation that there exists $\nu \in X^{\prime} \cap \mathcal{M}_{+}^{1}\left(\mathbb{R}^{d}\right)$ solution of $\left(\mathcal{P}^{*}\right)$ such that

$$
\sum_{i=1}^{p} \frac{\lambda_{i}}{2} W_{2}^{2}\left(\nu_{i}, \nu\right)=\sum_{i=1}^{p} \int_{\mathbb{R}^{d}} S_{\lambda_{i}} f_{i} d \nu_{i}=\sum_{i=1}^{p} \int_{\mathbb{R}^{d}} S_{\lambda_{i}} f_{i} d \nu_{i}+\sum_{i=1}^{p} \int_{\mathbb{R}^{d}} f_{i} d \nu .
$$

And since $\lambda_{i} W_{2}^{2}\left(\nu_{i}, \nu\right) / 2 \geq \int_{\mathbb{R}^{d}} S_{\lambda_{i}} f_{i} d \nu_{i}+\int_{\mathbb{R}^{d}} f_{i} d \nu$ because of (2.6), then (3.1) is equivalent to

$$
\frac{\lambda_{i}}{2} W_{2}^{2}\left(\nu_{i}, \nu\right)=\int_{\mathbb{R}^{d}} S_{\lambda_{i}} f_{i} d \nu_{i}+\int_{\mathbb{R}^{d}} f_{i} d \nu, \forall i \in\{1, \ldots, p\} .
$$

Now, let $\gamma_{i} \in \Pi\left(\nu_{i}, \nu\right)$ be an optimal transportation plan between $\nu_{i}$ and $\nu$ (i.e. $\gamma_{i} \in \Pi\left(\nu_{i}, \nu\right)$ and $\left.W_{2}^{2}\left(\nu_{i}, \nu\right)=\int_{\mathbb{R}^{d} \times \mathbb{R}^{d}}|x-y|^{2} d \gamma_{i}(x, y)\right)$. We deduce from (3.2) and $\gamma_{i} \in \Pi\left(\nu_{i}, \nu\right)$ that

$$
\begin{array}{r}
\frac{\lambda_{i}}{2} W_{2}^{2}\left(\nu_{i}, \nu\right)=\frac{\lambda_{i}}{2} \int_{\mathbb{R}^{d} \times \mathbb{R}^{d}}|x-y|^{2} d \gamma_{i}(x, y)=\int_{\mathbb{R}^{d}} S_{\lambda_{i}} f_{i} d \nu_{i}+\int_{\mathbb{R}^{d}} f_{i} d \nu \\
=\int_{\mathbb{R}^{d} \times \mathbb{R}^{d}}\left(S_{\lambda_{i}} f_{i}(x)+f_{i}(y)\right) d \gamma_{i}(x, y) .
\end{array}
$$

But since by definition of $S_{\lambda_{i}} f_{i}(x), \frac{\lambda_{i}}{2}|x-y|^{2} \geq S_{\lambda_{i}} f_{i}(x)+f_{i}(y)$, we get:

$$
\frac{\lambda_{i}}{2}|x-y|^{2}=S_{\lambda_{i}} f_{i}(x)+f_{i}(y), \quad \gamma_{i} \text {-a.e. }
$$

We have already noticed that $S_{\lambda_{i}}\left(S_{\lambda_{i}} f_{i}\right) \geq f_{i}$. So (3.3) implies that for $\gamma_{i^{-}}$a.e. $(x, y)$, one has $f_{i}(y)=\frac{\lambda_{i}}{2}|x-y|^{2}-S_{\lambda_{i}} f(x) \geq S_{\lambda_{i}}\left(S_{\lambda_{i}} f_{i}\right)(y)$, so that $f_{i}=S_{\lambda_{i}}\left(S_{\lambda_{i}} f_{i}\right) \nu$-a.e.. Therefore using the constraint $\sum_{i=1}^{p} f_{i}=0$ of $\left(\mathcal{P}^{*}\right)$, we have

$$
\sum_{i=1}^{p} S_{\lambda_{i}}\left(S_{\lambda_{i}} f_{i}\right) \geq 0, \sum_{i=1}^{p} S_{\lambda_{i}}\left(S_{\lambda_{i}} f_{i}\right)=0 \quad \nu \text {-a.e. }
$$


Now, we consider the convex function $\phi_{i}$ defined by

$$
\lambda_{i} \phi_{i}(x):=\frac{\lambda_{i}}{2}|x|^{2}-S_{\lambda_{i}} f_{i}(x),
$$

and we denote by $\partial \phi_{i}$ the graph of its subdifferential, i.e.

$$
\partial \phi_{i}:=\left\{(x, y) \in \mathbb{R}^{d} \times \mathbb{R}^{d}: \phi_{i}(x)+\phi_{i}^{*}(y)=x \cdot y\right\},
$$

where $\phi_{i}^{*}$ denotes the conjugate of $\phi_{i}$, i.e.

$$
\lambda_{i} \phi_{i}^{*}(y):=\frac{\lambda_{i}}{2}|y|^{2}-S_{\lambda_{i}}\left(S_{\lambda_{i}} f_{i}\right)(y)
$$

Combining (3.3) and (3.4), we have that the support of $\gamma_{i}$ is included in $\partial \phi_{i}$, and

$$
\sum_{i=1}^{p} \lambda_{i} \phi_{i}^{*}(y) \leq \frac{|y|^{2}}{2}, \forall y \in \mathbb{R}^{d}, \text { with equality } \nu \text {-a.e. }
$$

Moreover, it is easy to check that $\phi_{i}$ solves

$$
\inf \left\{\int_{\mathbb{R}^{d}} \phi(x) d \nu_{i}(x)+\int_{\mathbb{R}^{d}} \phi^{*}(y) d \nu(y): \phi \text { convex l.s.c. }\right\} .
$$

The previous conditions are of course very related to classical results from optimal transportation theory. To be more precise, let us first recall some definitions.

Definition 3.1. Let $\mu \in X^{\prime} \cap \mathcal{M}_{+}^{1}\left(\mathbb{R}^{d}\right)$ and $\sigma$ be a Borel map $\mathbb{R}^{d} \rightarrow \mathbb{R}^{d}$. The push-forward of $\mu$ through $\sigma$ is the measure denoted $\sigma \sharp \mu$ defined by

$$
\int_{\mathbb{R}^{d}} f(y) d(\sigma \sharp \mu)(y)=\int_{\mathbb{R}^{d}} f(\sigma(x)) d \mu(x), \forall f \in C_{b}\left(\mathbb{R}^{d}\right) .
$$

Definition 3.2. A probability measure $\mu \in X^{\prime} \cap \mathcal{M}_{+}^{1}\left(\mathbb{R}^{d}\right)$ is said to vanish on small sets if and only if $\mu(A)=0$ for every Borel set $A$ of $\mathbb{R}^{d}$, having Hausdorff dimension less than or equal to $d-1$.

Let us now recall the following classical results from optimal transportation theory which initially are due to Brenier and later generalized by McCann (see [3], [10] or [15]):

Proposition 3.3. Let $\mu$ and $\nu$ be in $X^{\prime} \cap \mathcal{M}_{+}^{1}\left(\mathbb{R}^{d}\right)$, and $\gamma \in \Pi(\mu, \nu)$. Then $\gamma$ is an optimal transportation plan between $\mu$ and $\nu$ if and only if the support of $\gamma$ is included in $\partial \phi$ for some convex l.s.c. function $\phi$; in this case $\phi$ solves

$$
\inf \left\{\int_{\mathbb{R}^{d}} \phi(x) d \mu(x)+\int_{\mathbb{R}^{d}} \phi^{*}(y) d \nu(y): \phi \text { convex l.s.c. }\right\} .
$$


If in addition $\mu$ vanishes on small sets, then there is a unique optimal transportation plan $\gamma$ which is of the form $\gamma=(\mathrm{id}, \nabla \phi) \sharp \mu$ with $\phi$ convex. Uniqueness also holds in the sense that if $\nabla \psi \sharp \mu=\nabla \phi \sharp \mu=\nu$ and $\psi$ is convex, then $\nabla \psi=\nabla \phi \mu$-almost everywhere.

Definition 3.4. Let $\mu$ and $\nu$ be in $X^{\prime} \cap \mathcal{M}_{+}^{1}\left(\mathbb{R}^{d}\right)$ such that $\mu$ vanishes on small sets. Then the Brenier map transporting $\mu$ to $\nu$ is the unique (up to $\mu$-a.e. equivalence) map of the form $\nabla \phi$ with $\phi$ convex such that $\nu=\nabla \phi \sharp \mu$.

Note that although Brenier's map $\nabla \phi$ is uniquely defined $\mu$-a.e., the potential $\phi$ is not (for instance, it is easy to build counter-examples if the support of $\mu$ is disconnected).

Thanks to the duality between $(\mathcal{P})$ and $\left(\mathcal{P}^{*}\right)$ and the extremality relations between these variational problems, we then deduce the following uniqueness result and characterization of the barycenter:

Proposition 3.5. Assume that there is an index $i \in\{1, \ldots p\}$ such that $\nu_{i}$ vanishes on small sets. Then $(\mathcal{P})$ admits a unique solution $\nu$ which is given by $\nu=\nabla \phi_{i} \sharp \nu_{i}$ where $\phi_{i}$ is the convex potential defined by (3.5).

Proof. Let $\nu$ solve $(\mathcal{P})$, let $\left(\phi_{j}\right)_{j=1, \ldots, p}$ be the convex potentials defined by (3.5) (these potentials do not depend on $\nu$ but only on a solution to the dual problem $\left.\left(\mathcal{P}^{*}\right)\right)$ and let $\gamma_{i} \in \Pi\left(\nu_{i}, \nu\right)$ be an optimal transport plan between $\nu_{i}$ and $\nu$. Thanks to (3.3)-(3.4), we know that the support of $\gamma_{i}$ is included in the graph of $\partial \phi_{i}$. Since $\nu_{i}$ vanishes on small sets, $\phi_{i}$ is differentiable $\nu_{i}$-almost everywhere so that $\gamma_{i}=\left(\mathrm{id}, \nabla \phi_{i}\right) \sharp \nu_{i}$ which implies that $\nu=\nabla \phi_{i} \sharp \nu_{i}$ and this determines $\nu$ uniquely.

This enables us to define unambiguously barycenters as follows:

Definition 3.6. Given $\left(\nu_{1}, \ldots, \nu_{p}\right)$ in $X^{\prime} \cap \mathcal{M}_{+}^{1}\left(\mathbb{R}^{d}\right)$ one of which vanishes on small sets, and given positive reals $\left(\lambda_{1}, \ldots, \lambda_{p}\right)$ that sum to 1 , the barycenter of $\left(\nu_{1}, \ldots, \nu_{p}\right)$ with weights $\left(\lambda_{1}, \ldots, \lambda_{p}\right)$ is the unique solution of $(\mathcal{P})$. It will be denoted by $\left(\operatorname{bar}\left(\nu_{i}, \lambda_{i}\right)_{i=1, \ldots, p}\right)$.

Remark 3.7. If all the $\nu_{i}$ 's vanish on small sets, defining $\phi_{i}$ by (3.5) (with $\left(f_{1}, \ldots, f_{p}\right)$ an arbitrary solution of $\left.\left(\mathcal{P}^{*}\right)\right)$ we see that $\nu=\nabla \phi_{i} \sharp \nu_{i}$ for every $i$. The condition that $\nabla \phi_{i} \sharp \nu_{i}$ does not depend on $i$ then appears as an optimality condition for $\left(\mathcal{P}^{*}\right)$.

The following proposition further characterizes the barycenter.

Proposition 3.8. Assume that $\nu_{i}$ vanishes on small sets for every $i=1, \ldots, p$, and let $\nu \in X^{\prime} \cap \mathcal{M}_{+}^{1}\left(\mathbb{R}^{d}\right)$. Then the following conditions are equivalent: 
1. $\nu$ solves $(\mathcal{P})$.

2. $\nu=\nabla \phi_{i} \sharp \nu_{i}$ for every $i$, where $\phi_{i}$ is defined by (3.5).

3. There exist convex potentials $\psi_{i}$ such that $\nabla \psi_{i}$ is Brenier's map transporting $\nu_{i}$ to $\nu$, and a constant $C$ such that

$$
\sum_{i=1}^{p} \lambda_{i} \psi_{i}^{*}(y) \leq C+\frac{|y|^{2}}{2}, \forall y \in \mathbb{R}^{d} \text {, with equality } \nu \text {-a.e. }
$$

Proof. The equivalence between 1. and 2. follows from proposition 3.5. Next we prove that 1 . is equivalent to 3 . Indeed, if 1 . holds, then it is enough to take $\psi_{i}=\phi_{i}$ where $\phi_{i}$ is defined by (3.5); then (3.9) directly follows from (3.6). Finally, if 3. is satisfied (with $C=0$, say), then (3.9) together with the Kantorovich duality formula gives

$$
\sum_{i=1}^{p} \frac{\lambda_{i}}{2} W_{2}^{2}\left(\nu_{i}, \nu\right)=\sum_{i=1}^{p} \lambda_{i} \int_{\mathbb{R}^{d}}\left(\frac{|x|^{2}}{2}-\psi_{i}(x)\right) d \nu_{i}(x) .
$$

Now let $\mu \in X^{\prime} \cap \mathcal{M}_{+}^{1}\left(\mathbb{R}^{d}\right)$. Using the inequality $x \cdot y \leq \psi_{i}(x)+\psi_{i}^{*}(y)$, i.e.

$$
\lambda_{i} \frac{|x|^{2}}{2}-\lambda_{i} \psi_{i}(x)+\lambda_{i} \frac{|y|^{2}}{2}-\lambda_{i} \psi_{i}^{*}(y) \leq \frac{\lambda_{i}}{2}|x-y|^{2},
$$

we have after integration

$$
\frac{\lambda_{i}}{2} W_{2}^{2}\left(\nu_{i}, \mu\right) \geq \lambda_{i} \int_{\mathbb{R}^{d}}\left(\frac{|x|^{2}}{2}-\psi_{i}(x)\right) d \nu_{i}(x)+\lambda_{i} \int_{\mathbb{R}^{d}}\left(\frac{|y|^{2}}{2}-\psi_{i}^{*}(y)\right) d \mu(y) .
$$

Summing these inequalities over $i$, and using (3.9), we get

$$
\begin{aligned}
\sum_{i=1}^{p} \frac{\lambda_{i}}{2} W_{2}^{2}\left(\nu_{i}, \mu\right) & \geq \sum_{i=1}^{p} \lambda_{i} \int_{\mathbb{R}^{d}}\left(\frac{|x|^{2}}{2}-\psi_{i}(x)\right) d \nu_{i}(x)+\int_{\mathbb{R}^{d}}\left(\frac{|y|^{2}}{2}-\sum_{i=1}^{p} \lambda_{i} \psi_{i}^{*}(y)\right) d \mu(y) \\
& \geq \sum_{i=1}^{p} \lambda_{i} \int_{\mathbb{R}^{d}}\left(\frac{|x|^{2}}{2}-\psi_{i}(x)\right) d \nu_{i}(x)=\sum_{i=1}^{p} \frac{\lambda_{i}}{2} W_{2}^{2}\left(\nu_{i}, \nu\right)
\end{aligned}
$$

which proves that $\nu$ solves $(\mathcal{P})$.

Remark 3.9. If $\nu$ and the potentials $\psi_{i}$ satisfy the third statement of the previous proposition, then the support of $\nu$-almost every point is included in the contact set where the convex function $\varphi:=\sum_{i=1}^{p} \lambda_{i} \psi_{i}^{*}$ agrees with its quadratic majorant $C+\frac{|.|^{2}}{2}$. Since these two functions are continuous, this 
implies that the support of $\nu, \operatorname{Supp}(\nu)$ is actually included in this contact set. Now let us remark that at such a contact point $x$, we have

$$
\sum_{i=1}^{p} \lambda_{i} \partial \psi_{i}^{*}(x)=\left\{\sum_{i=1}^{p} \lambda_{i} q_{i}, q_{i} \in \partial \psi_{i}^{*}(x)\right\} \subset \partial \varphi(x) \subset\{x\}
$$

so that each potential $\psi_{i}^{*}$ is differentiable at $x$. The potentials $\psi_{i}^{*}$ are therefore differentiable on $\operatorname{Supp}(\nu)$ and satisfy on this set the relation

$$
\sum_{i=1}^{p} \lambda_{i} \nabla \psi_{i}^{*}=\mathrm{id}
$$

We also remark that if (3.10) holds everywhere for Brenier's maps $\nabla \psi_{i}$ transporting $\nu_{i}$ to $\nu$, then $\nu$ is optimal for $(\mathcal{P})$.

\section{Multi-marginal formulation}

Our aim in this section is to prove that $(\mathcal{P})$ is equivalent to a linear programming problem of multi-marginal optimal transportation type similar to the one solved by Gangbo and Świȩch in [8].

For every $x:=\left(x_{1}, \ldots, x_{p}\right) \in\left(\mathbb{R}^{d}\right)^{p}$, we define

$$
T(x):=\sum_{i=1}^{p} \lambda_{i} x_{i}
$$

Of course the Euclidean barycenter $T(x)$ is characterized by the property

$$
\sum_{i=1}^{p} \lambda_{i}\left|x_{i}-T(x)\right|^{2}=\inf _{y \in \mathbb{R}^{d}}\left\{\sum_{i=1}^{p} \lambda_{i}\left|x_{i}-y\right|^{2}\right\}
$$

Let us now introduce the multi-marginal optimal transportation problem

$$
\inf \left\{\int_{\left(\mathbb{R}^{d}\right)^{p}}\left(\sum_{i=1}^{p} \frac{\lambda_{i}}{2}\left|x_{i}-T(x)\right|^{2}\right) d \gamma\left(x_{1}, \ldots x_{p}\right), \gamma \in \Pi\left(\nu_{1}, \ldots, \nu_{p}\right)\right\}
$$

where $\Pi\left(\nu_{1}, \ldots, \nu_{p}\right)$ is the set of probability measures on $\left(\mathbb{R}^{d}\right)^{p}$ having $\nu_{1}, \ldots, \nu_{p}$ as marginals. Developing the squares in (4.3), it is easy to see that (4.3) is equivalent to

$$
(\mathcal{Q}) \sup \left\{\int_{\left.\mathbb{R}^{d}\right)^{p}}\left(\sum_{1 \leq i<j \leq p} \lambda_{i} \lambda_{j} x_{i} \cdot x_{j}\right) d \gamma\left(x_{1}, \ldots x_{p}\right), \gamma \in \Pi\left(\nu_{1}, \ldots, \nu_{p}\right)\right\} .
$$


The previous multi-marginal problem $(\mathcal{Q})$ has been solved by Gangbo and Święch in [8]. As usual, a key tool is the dual problem

$$
\left(\mathcal{Q}^{*}\right) \inf \left\{\sum_{i=1}^{p} \int_{\mathbb{R}^{d}} g_{i} d \nu_{i}, \sum_{i=1}^{p} g_{i}\left(x_{i}\right) \geq \sum_{1 \leq i<j \leq p} \lambda_{i} \lambda_{j} x_{i} \cdot x_{j}, \forall x \in\left(\mathbb{R}^{d}\right)^{p}\right\} .
$$

As the classical optimal transportation problem with quadratic cost is solved by Brenier, $(\mathcal{Q})$ and $\left(\mathcal{Q}^{*}\right)$ have a very special structure that we next discuss heuristically. Firstly, in $\left(\mathcal{Q}^{*}\right)$, one can restrict to potentials that satisfy

$$
g_{i}\left(x_{i}\right)=\sup _{\left(x_{j}\right)_{j \neq i}}\left\{\frac{1}{2} \sum_{1 \leq k \neq j \leq p} \lambda_{k} \lambda_{j} x_{k} \cdot x_{j}-\sum_{j \neq i} g_{j}\left(x_{j}\right)\right\}
$$

which, in particular, implies the convexity of the potentials $g_{i}$ 's. Secondly, the duality relation between $(\mathcal{Q})$ and $\left(\mathcal{Q}^{*}\right)$ expresses that, $\gamma$ solves $(\mathcal{Q})$ and $\left(g_{1}, \ldots, g_{p}\right)$ solves $\left(\mathcal{Q}^{*}\right)$ if and only if

$$
\sum_{i=1}^{p} g_{i}\left(x_{i}\right)=\frac{1}{2} \sum_{1 \leq i \neq j \leq p} \lambda_{i} \lambda_{j} x_{i} \cdot x_{j}, \gamma \text {-a.e.. }
$$

Finally, if, in addition the potentials $g_{i}$ 's are differentiable $\gamma$-a.e., then one can deduce from (4.6) and (4.7) that for $\gamma$-a.e. $x=\left(x_{1}, \ldots, x_{d}\right)$, one has

$$
\nabla g_{i}\left(x_{i}\right)=\lambda_{i} \sum_{j \neq i} \lambda_{j} x_{j}
$$

which can be rewritten as

$$
\nabla\left(\frac{\lambda_{i}}{2}|\cdot|^{2}+\frac{g_{i}}{\lambda_{i}}\right)\left(x_{i}\right)=\sum_{j=1}^{p} \lambda_{j} x_{j}=\nabla\left(\frac{\lambda_{1}}{2}|\cdot|^{2}+\frac{g_{1}}{\lambda_{1}}\right)\left(x_{1}\right)
$$

or in a more explicit way

$$
x_{i}=\nabla\left(\frac{\lambda_{i}}{2}|\cdot|^{2}+\frac{g_{i}}{\lambda_{i}}\right)^{*} \circ \nabla\left(\frac{\lambda_{1}}{2}|\cdot|^{2}+\frac{g_{1}}{\lambda_{1}}\right)\left(x_{1}\right) .
$$

This (formally) yields that the optimal $\gamma$ is in fact supported by the graph of a map of the form $x_{1} \rightarrow\left(x_{1}, \nabla u_{2}^{*}\left(\nabla u_{1}\left(x_{1}\right)\right), \ldots, \nabla u_{p}^{*}\left(\nabla u_{1}\left(x_{1}\right)\right)\right)$ for some potentials $u_{i}$ such that $u_{i}-\lambda_{i}|.|^{2} / 2$ is convex. The previous discussion being informal, we refer to the paper of Gangbo and Święch [8] for the details. Here, we simply summarize their main results as follows: 
Theorem 4.1. Assume that $\nu_{i}$ vanishes on small sets for $i=1, \ldots, p$. Then $(\mathcal{Q})$ admits a unique solution $\gamma \in \Pi\left(\nu_{1}, \ldots, \nu_{p}\right)$. Moreover, $\gamma$ is of the form $\gamma=\left(T_{1}^{1}, \ldots, T_{p}^{1}\right) \sharp \nu_{1}$ with $T_{i}^{1}=\nabla u_{i}^{*} \circ \nabla u_{1}$ for $i=1, \ldots, p$ where the $u_{i}$ 's are strictly convex potentials defined by

$$
u_{i}(x):=\frac{\lambda_{i}}{2}|x|^{2}+\frac{g_{i}(x)}{\lambda_{i}}, \forall x \in \mathbb{R}^{d}
$$

and $\left(g_{1}, \ldots, g_{p}\right)$ are convex potentials that solve $\left(\mathcal{Q}^{*}\right)$.

In the sequel, we will refer to the maps $T_{i}^{1}$ of the previous theorem as the Gangbo-Święch maps between $\nu_{1}$ and $\nu_{i}$. Note that the Gangbo-Święch maps a priori depend on the whole collections of the $\nu_{i}$ 's and the weights $\lambda_{i}$ 's. These maps are transport maps in the sense that $T_{i}^{1} \sharp \nu_{1}=\nu_{i}$. Of course, by permuting the indices, one can similarly define the Gangbo-Święch maps $T_{i}^{j}:=\nabla u_{i}^{*} \circ \nabla u_{j}$ between a reference measure $\nu_{j}$ and $\nu_{i}$.

The next result gives the precise relationship between our initial barycenter problem $(\mathcal{P})$ and the multi-marginals problem $(\mathcal{Q})$.

Proposition 4.2. Assume that $\nu_{i}$ vanishes on small sets for $i=1, . ., p$. Then the solution of $(\mathcal{P})$ is given by $\nu=T \sharp \gamma$, where $T$ is defined by (4.1) and $\gamma$ is the solution of $(\mathcal{Q})$.

Proof. For every $i \in\{1, \ldots, p\}$ denote by $\pi_{i}$ the $i$-th canonical projection from $\left(\mathbb{R}^{d}\right)^{p}$ to $\mathbb{R}^{d}$ (i.e. $\left.\pi_{i}(x)=x_{i}\right)$ and define $\gamma_{i}:=\left(\pi_{i}, T\right) \sharp \gamma$. By construction, $\gamma_{i} \in \Pi\left(\nu_{i}, \nu\right)$. Then

$$
W_{2}^{2}\left(\nu_{i}, \nu\right) \leq \int_{\left(\mathbb{R}^{d}\right)^{p}}\left|x_{i}-T(x)\right|^{2} d \gamma(x)
$$

and thus,

$$
\sum_{i=1}^{p} \frac{\lambda_{i}}{2} W_{2}^{2}\left(\nu_{i}, \nu\right) \leq \int_{\left(\mathbb{R}^{d}\right)^{p}}\left(\sum_{i=1}^{p} \frac{\lambda_{i}}{2}\left|x_{i}-T(x)\right|^{2}\right) d \gamma(x) .
$$

Now let $\mu \in X^{\prime} \cap \mathcal{M}_{+}^{1}\left(\mathbb{R}^{d}\right)$ and $\eta_{i} \in \Pi\left(\nu_{i}, \mu\right)$ for $i=1, \ldots, p$. By the disintegration theorem (see [6]), we can write $\eta_{i}=\eta_{i}^{y} \otimes \mu$ for a Borel family of (conditional) probability measures $\left(\eta_{i}^{y}\right)_{y \in \mathbb{R}^{d}}$ which precisely means that

$\int_{\mathbb{R}^{d} \times \mathbb{R}^{d}} f\left(x_{i}, y\right) d \eta_{i}\left(x_{i}, y\right)=\int_{\mathbb{R}^{d}}\left(\int_{\mathbb{R}^{d}} f\left(x_{i}, y\right) d \eta_{i}^{y}\left(x_{i}\right)\right) d \mu(y), \forall f \in C_{b}^{0}\left(\mathbb{R}^{d} \times \mathbb{R}^{d}\right)$.

Now, let us define $\eta \in \mathcal{M}_{+}^{1}\left(\left(\mathbb{R}^{d}\right)^{p+1}\right)$ by

$$
\int_{\left(\mathbb{R}^{d}\right)^{p+1}} f(x, y) d \eta(x, y)=\int_{\mathbb{R}^{d}}\left(\int_{\left(\mathbb{R}^{d}\right)^{p}} f(x, y) d \eta_{1}^{y}\left(x_{1}\right) \ldots d \eta_{p}^{y}\left(x_{p}\right)\right) d \mu(y)
$$


for every test-function $f \in C_{b}\left(\left(\mathbb{R}^{d}\right)^{p+1}\right)$. Let $\theta \in \mathcal{M}_{+}^{1}\left(\left(\mathbb{R}^{d}\right)^{p}\right)$ be the canonical projection of $\eta$ on $\left(\mathbb{R}^{d}\right)^{p}$ i.e.

$\int_{\left(\mathbb{R}^{d}\right)^{p}} f(x) d \theta(x)=\int_{\mathbb{R}^{d}}\left(\int_{\left(\mathbb{R}^{d}\right)^{p}} f(x) d \eta_{1}^{y}\left(x_{1}\right) \ldots d \eta_{p}^{y}\left(x_{p}\right)\right) d \mu(y), \forall f \in C_{b}^{0}\left(\left(\mathbb{R}^{d}\right)^{p}\right)$.

By construction, $\theta \in \Pi\left(\nu_{1}, \ldots, \nu_{p}\right)$. Using (4.2) and the fact that $\gamma$ solves (4.3), we then obtain

$$
\begin{aligned}
\sum_{i=1}^{p} \frac{\lambda_{i}}{2} \int_{\mathbb{R}^{d} \times \mathbb{R}^{d}}\left|x_{i}-y\right|^{2} d \eta_{i}\left(x_{i}, y\right) & =\int_{\left(\mathbb{R}^{d}\right)^{p+1}}\left(\sum_{i=1}^{p} \frac{\lambda_{i}}{2}\left|x_{i}-y\right|^{2}\right) d \eta(x, y) \\
& \geq \int_{\left(\mathbb{R}^{d}\right)^{p+1}}\left(\sum_{i=1}^{p} \frac{\lambda_{i}}{2}\left|x_{i}-T(x)\right|^{2}\right) d \eta(x, y) \\
& =\int_{\left(\mathbb{R}^{d}\right)^{p}}\left(\sum_{i=1}^{p} \frac{\lambda_{i}}{2}\left|x_{i}-T(x)\right|^{2}\right) d \theta(x) \\
& \geq \int_{\left(\mathbb{R}^{d}\right)^{p}}\left(\sum_{i=1}^{p} \frac{\lambda_{i}}{2}\left|x_{i}-T(x)\right|^{2}\right) d \gamma(x)
\end{aligned}
$$

Since in the previous inequality, the $\eta_{i}$ 's are arbitrary transport plans between $\nu_{i}$ and $\mu$, we conclude with (4.9).

Combining Theorem 4.1 and Proposition 4.2, we see that the barycenter of the $\left(\nu_{i}, \lambda_{i}\right)$ 's is also characterized by

$$
\nu:=\left(\sum_{i=1}^{p} \lambda_{i} T_{i}^{1}\right) \sharp \nu_{1}=\left(\sum_{i=1}^{p} \lambda_{i} T_{i}^{j}\right) \sharp \nu_{j}
$$

where the $T_{i}^{j}$ are the Gangbo-Święch maps between $\nu_{j}$ and $\nu_{i}$ which are given by $T_{i}^{j}=\nabla u_{i}^{*} \circ \nabla u_{j}$, where the $u_{j}$ 's are strictly convex potentials defined by (4.8). As an immediate consequence, we deduce that the support of the barycenter $\operatorname{bar}\left(\left(\nu_{i}, \lambda_{i}\right)_{i}\right)$ is included in $\sum_{i=1}^{p} \lambda_{i} \operatorname{Supp}\left(\nu_{i}\right)$. Let us also remark that the center of mass of $\operatorname{bar}\left(\left(\nu_{i}, \lambda_{i}\right)_{i}\right)$ is $\sum_{i=1}^{p} \lambda_{i} \int_{\mathbb{R}^{d}} x d \nu_{i}(x)$.

\section{Regularity of the barycenter}

The relation between the barycenter and the Gangbo-Święch maps easily enables us to obtain a regularity result on the barycenter. 
Theorem 5.1. Let $\left(\nu_{1}, \ldots, \nu_{p}\right)$ in $X^{\prime} \cap \mathcal{M}_{+}^{1}\left(\mathbb{R}^{d}\right)$ vanish on small sets and let $\left(\lambda_{1}, \ldots, \lambda_{p}\right)$ be positive reals that sum to 1 . Also, assume that $\nu_{1} \in L^{\infty}$ (i.e. is absolutely continuous and has a bounded density with respect to the Lebesgue measure) and define $\bar{\nu}:=\operatorname{bar}\left(\left(\nu_{i}, \lambda_{i}\right)\right)$. Then $\bar{\nu} \in L^{\infty}$ and more precisely:

$$
\|\bar{\nu}\|_{L^{\infty}} \leq \frac{1}{\lambda_{1}^{d}}\left\|\nu_{1}\right\|_{L^{\infty}} .
$$

Proof. By proposition $4.2, \bar{\nu}=\bar{T} \sharp \nu_{1}$ where

$$
\bar{T}=\sum_{i=1}^{p} \lambda_{i} \nabla u_{i}^{*} \circ \nabla u_{1}=\lambda_{1} \mathrm{id}+\sum_{i=2}^{p} \lambda_{i} \nabla u_{i}^{*} \circ \nabla u_{1}
$$

and the potentials $u_{i}$ are defined as in the previous section, in particular each $u_{i}$ is strictly convex and more precisely $u_{i}-\frac{\lambda_{i}}{2}||^{2}$ is convex so that one has in the sense of distributions

$$
D^{2} u_{i} \geq \lambda_{i} \text { id, }
$$

(given two symmetric matrices $A$ and $B$, the notation $A \geq B$ means, as usual, that $A-B$ is semi-definite positive). Since $u_{i}-\frac{\lambda_{i}}{2}|\cdot|^{2}$ is convex, $u_{i}^{*}$ is $C^{1}$ and for every $p$ and $q$ in $\mathbb{R}^{d}$, since $p \in \partial u_{i}\left(\nabla u_{i}^{*}(p)\right)$ and $q \in \partial u_{i}\left(\nabla u_{i}^{*}(q)\right)$, we have

$$
\left.\left.\left(\nabla u_{i}^{*}(p)\right)-\nabla u_{i}^{*}(q)\right) \cdot(p-q) \geq \lambda_{i} \mid \nabla u_{i}^{*}(p)\right)-\left.\nabla u_{i}^{*}(q)\right|^{2}
$$

which shows that $\nabla u_{i}^{*}$ is $\lambda_{i}^{-1}$-Lipschitz, so that $u_{i}^{*} \in C^{1,1}$, and

$$
D^{2} u_{i}^{*} \leq \frac{1}{\lambda_{i}} \mathrm{id} .
$$

For $\varepsilon>0$, we regularize $u_{1}$ as follows:

$$
u_{1}^{\varepsilon}:=\rho_{\varepsilon} \star v_{1}^{\varepsilon}
$$

where $\rho_{\varepsilon}:=\varepsilon^{-d} \rho(. / \varepsilon), \rho \in C_{c}^{\infty}, \rho \geq 0, \int \rho=1$ and $\operatorname{Supp}(\rho) \subset B_{1}$, and we define $v_{1}^{\varepsilon}$ as the usual Moreau-Yosida regularization by infimal-convolution:

$$
v_{1}^{\varepsilon}:=\left(u_{1}^{*}+\frac{\varepsilon}{2}|\cdot|^{2}\right)^{*} .
$$

By construction, $u_{1}^{\varepsilon}$ is smooth and strictly convex, and by the same arguments as above, we precisely have

$$
\varepsilon \text { id } \leq D^{2}\left(u_{1}^{\varepsilon}\right)^{*} \leq\left(\frac{1}{\lambda_{1}}+\varepsilon\right) \text { id, } \varepsilon^{-1} \text { id } \geq D^{2} u_{1}^{\varepsilon} \geq\left(\frac{\lambda_{1}}{1+\lambda_{1} \varepsilon}\right) \text { id } .
$$


Moreover, it is classical to check that $\nabla u_{1}^{\varepsilon}$ converges to $\nabla u_{1}$ in $L_{\text {loc }}^{1}$. We can now define the Lipschitz map

$$
\bar{T}^{\varepsilon}=\lambda_{1} \mathrm{id}+\sum_{i=2}^{p} \lambda_{i} \nabla u_{i}^{*} \circ \nabla u_{1}^{\varepsilon}
$$

and the image measure $\bar{\nu}^{\varepsilon}:=\bar{T}^{\varepsilon} \sharp \nu_{1}$. We first claim that $\bar{T}^{\varepsilon}$ is injective. Indeed, if $0=\bar{T}^{\varepsilon}(x)-\bar{T}^{\varepsilon}(y)$, taking the scalar product with $\nabla u_{1}^{\varepsilon}(x)-\nabla u_{1}^{\varepsilon}(y)$ and using the monotonicity of $\nabla u_{i}^{*}$, we get

$$
\begin{aligned}
0 & =\lambda_{1}(x-y) \cdot\left(\nabla u_{1}^{\varepsilon}(x)-\nabla u_{1}^{\varepsilon}(y)\right) \\
& +\sum_{i=2}^{p} \lambda_{i}\left(\nabla u_{i}^{*}\left(\nabla u_{1}^{\varepsilon}(x)\right)-\nabla u_{i}^{*}\left(\nabla u_{1}^{\varepsilon}(y)\right)\right) \cdot\left(\nabla u_{1}^{\varepsilon}(x)-\nabla u_{1}^{\varepsilon}(y)\right) \\
& \geq \frac{\lambda_{1}^{2}}{1+\lambda_{1} \varepsilon}|x-y|^{2}
\end{aligned}
$$

which proves that $x=y$. The same argument applies to show that $\bar{T}$ is also injective when restricted to the set of points where $u_{1}$ is differentiable.

Since $\nabla u_{1}^{\varepsilon}$ is a smooth diffeomorphism with Jacobian bounded away from zero, and since the maps $\nabla u_{i}^{*}$ are Lipschitz, the singular set $S$ of points $x$ such that $\sum_{i=2}^{p} \lambda_{i} \nabla u_{i}^{*}$ fails to be differentiable at $\nabla u_{1}^{\varepsilon}(x)$ is Lebesgue negligible. For $x \notin S$, by the usual chain rule, we have

$$
D \bar{T}^{\varepsilon}(x)=\lambda_{1} \mathrm{id}+\sum_{i=2}^{p} \lambda_{i} D^{2} u_{i}^{*}\left(\nabla u_{1}^{\varepsilon}(x)\right) D^{2} u_{1}^{\varepsilon}(x)
$$

which we rewrite as $\lambda_{1}$ id $+A B$ where both matrices $A:=D^{2} u_{i}^{*}\left(\nabla u_{1}^{\varepsilon}(x)\right)$ and $B:=D^{2} u_{1}^{\varepsilon}(x)$ are symmetric and positive definite. Now, we claim that this implies

$$
\operatorname{det} D \bar{T}^{\varepsilon}(x)=\operatorname{det}\left(\lambda_{1} \mathrm{id}+A B\right) \geq \lambda_{1}^{d} .
$$

Indeed, if $z \in \mathbb{R}^{d}$ and $\mu \in \mathbb{R}$, we have $A B z=\mu z$ if and only if $\left(B^{1 / 2} A B^{1 / 2}\right) B^{1 / 2} z=\mu B^{1 / 2} z$. So $A B$ has a basis of eigenvectors (because $B^{1 / 2} A B^{1 / 2}$ has one and $B^{1 / 2}$ is invertible) and all its eigenvalues are positive since they are eigenvalues of the definite positive matrix $B^{1 / 2} A B^{1 / 2}$. Hence $\lambda_{1}$ id $+A B$ has a basis of eigenvectors and all its eigenvalues are larger than $\lambda_{1}$, which proves (5.5). By standard results (see for instance lemma 5.5.3 in [1]), we obtain that $\bar{\nu}^{\varepsilon} \in L^{\infty}$ and

$$
\left\|\bar{\nu}^{\varepsilon}\right\|_{L^{\infty}} \leq \frac{1}{\lambda_{1}^{d}}\left\|\nu_{1}\right\|_{L^{\infty}} .
$$


We easily deduce (5.1) by remarking that for some sequence $\varepsilon_{n}$ converging to $0, \bar{\nu}_{\varepsilon_{n}}$ converges narrowly to $\bar{\nu}$ because $\bar{T}^{\varepsilon_{n}}$ converges a.e. to $\bar{T}$.

Remark 5.2. In the previous $L^{\infty}$ estimate, it is actually not necessary to require that all the measures $\nu_{i}$ vanish on small sets; this assumption just allowed us to use results of [8]. Indeed, if we simply assume that $\nu_{1} \in L^{\infty}$ vanishes on small sets, it follows from proposition 3.5 that $\bar{\nu}=\operatorname{bar}\left(\nu_{i}, \lambda_{i}\right)_{i}$ is uniquely defined. Moreover it is easy to check that if we approximate $\left(\nu_{i}\right)_{i=2, ., p}$ by measures that vanish on small sets, the barycenter of the approximations converges narrowly to $\bar{\nu}$. We thus recover the estimate (5.1) by approximation.

\section{Examples}

\subsection{The case $d=1$}

In the unidimensional space case, $d=1$, the description of the barycenter is quite simple and this is (roughly speaking) due to the fact that gradient of convex functions are simply nondrecreasing functions, and this property is stable by composition. Let $\nu_{1}, \ldots, \nu_{p}$ be nonatomic probability measures on the real line that have finite second moments, and let $\lambda_{1}, \ldots, \lambda_{p}$ be positive reals that sum to 1 . From formula (4.10), the barycenter $\bar{\nu}:=\operatorname{bar}\left(\nu_{i}, \lambda_{i}\right)_{i}$ is given by

$$
\bar{\nu}=\left(\sum_{i=1} \lambda_{i} T_{i}^{1}\right) \sharp \nu_{1}
$$

where $T_{i}^{1}$ is the Gangbo-Święch map between $\nu_{1}$ and $\nu_{i}$. Therefore, $T_{i}^{1}$ is a nondecreasing map that pushes $\nu_{1}$ forward to $\nu_{i}$. There is only one such map, and that is of course the Brenier map which is given by the usual rearrangement or quantile-like formula $T_{i}^{1}:=F_{i}^{-1} \circ F_{1}$, where $F_{i}$ is the cumulative function of $F_{i}$ i.e. $F_{i}(\alpha)=\nu_{i}((-\infty, \alpha])$, and $F_{i}^{-1}$ denotes the generalized inverse of $F_{i}$,

$$
F_{i}^{-1}(t):=\inf \left\{\alpha: F_{i}(\alpha) \geq t\right\} .
$$

Therefore, $\operatorname{bar}\left(\nu_{i}, \lambda_{i}\right)_{i}$ is simply obtained as the image of $\nu_{1}$ by the linearly interpolated transport map $\sum_{i} \lambda_{i} T_{i}^{1}$. Of course, one also has

$$
\bar{\nu}=\left(\sum_{i=1} \lambda_{i} T_{i}^{j}\right) \sharp \nu_{j}
$$


where $T_{i}^{j}$ is Brenier's map between $\nu_{j}$ and $\nu_{i}$. The fact that the resulting measure does not depend on $j$ is very specific to the unidimensional case and does not hold in general in higher dimensions.

\subsection{The case $p=2$}

In the case of two measures $\nu_{0}$ and $\nu_{1}$ (regular enough), and $t \in(0,1)$, it is reasonable to expect that the barycenter of $\left(\nu_{0},(1-t)\right)$ and $\left(\nu_{1}, t\right)$ is McCann's interpolant [11]

$$
\nu_{t}:=((1-t) \mathrm{id}+t \nabla \phi) \sharp \nu_{0}=\left(t \mathrm{id}+(1-t) \nabla \phi^{*}\right) \sharp \nu_{1}
$$

where $\nabla \phi$ is Brenier's map between $\nu_{0}$ and $\nu_{1}$. This is indeed an easy consequence of the triangle inequality but we would like to take advantage of this simple case to illustrate the duality characterization of proposition 3.8. To show that $\nu_{t}$ satisfies the optimality condition of proposition 3.8 it is enough to prove that

$$
(1-t) f_{t}+t g_{t}=\frac{1}{2}|\cdot|^{2}+C
$$

where $C$ is constant, and $\nabla f_{t}$ and $\nabla g_{t}$ are respectively Brenier's maps between $\nu_{t}$ and $\nu_{0}$, and $\nu_{t}$ and $\nu_{1}$, i.e.,

$$
f_{t}=\left(\frac{(1-t)}{2}|\cdot|^{2}+t \phi\right)^{*}, g_{t}=\left(\frac{t}{2}|\cdot|^{2}+(1-t) \phi^{*}\right)^{*} \text {. }
$$

To prove the identity (6.1) (with $C=0$ in fact), we first write

$$
-f_{t}(p)=\inf _{x \in \mathbb{R}^{d}}\left\{(1-t) \frac{|x|^{2}}{2}-p \cdot x+t \phi(x)\right\}
$$

which, thanks to the Fenchel-Rockafellar duality theorem, can be rewritten as

$$
-f_{t}(p)=\sup _{z \in \mathbb{R}^{d}}\left\{-t \phi^{*}(-z / t)-\frac{1}{2(1-t)}|p+z|^{2}\right\} .
$$

Therefore

$$
\begin{aligned}
-(1-t) f_{t}(p) & =\sup _{y \in \mathbb{R}^{d}}\left\{-t(1-t) \phi^{*}(y)-\frac{1}{2}|p-t y|^{2}\right\} \\
& =-\frac{1}{2}|p|^{2}+t \sup _{y \in \mathbb{R}^{d}}\left\{p \cdot y-\left(\frac{t}{2}|y|^{2}+(1-t) \phi^{*}(y)\right)\right\} \\
& =-\frac{1}{2}|p|^{2}+t g_{t}(p) .
\end{aligned}
$$

We thus conclude that the set of barycenters of $\nu_{0}$ and $\nu_{1}$ is nothing but the geodesic curve $t \in[0,1] \mapsto \nu_{t}$ given by McCann's interpolation [11]. 


\subsection{The gaussian case}

Let us now consider the case where for $i=1, \ldots, p, \nu_{i}=\mathcal{N}\left(0, S_{i}\right)$ i.e. $\nu_{i}$ is a gaussian measure with mean 0 and covariance matrix $S_{i}$. We assume that each $S_{i}$ is positive definite and, given weights $\lambda_{i}>0$ that sum to 1 , we consider again the barycenter problem (2.2). This gaussian case was already considered by Knott and Smith [9] who suggested an almost explicit construction for the barycenter (which turns out to be gaussian as well). But the existence and uniqueness of the barycenter was not proved in their paper. The following theorem addresses these issues and also gives an explicit construction of the barycenter in this case.

Theorem 6.1. In the gaussian framework of this paragraph, there is a unique solution $\bar{\nu}$ to (2.2). Moreover, $\bar{\nu}=\mathcal{N}(0, \bar{S})$ where $\bar{S}$ is the unique positive definite root of the matrix equation

$$
\sum_{i=1}^{p} \lambda_{i}\left(S^{1 / 2} S_{i} S^{1 / 2}\right)^{1 / 2}=S
$$

Proof. Step 1: existence of a solution to (6.2). Let $\alpha_{i}$ and $\beta_{i}$ denote respectively the smallest and largest eigenvalue of $S_{i}$, and $\alpha$ and $\beta$ be such that

$$
\beta \geq\left(\sum_{i=1}^{p} \lambda_{i} \sqrt{\beta_{i}}\right)^{2} \geq \sum_{i=1}^{p}\left(\lambda_{i} \sqrt{\alpha_{i}}\right)^{2} \geq \alpha
$$

Let $K_{\alpha, \beta}$ be the (convex and compact) set of symmetric matrices $S$ such that $\beta I \geq S \geq \alpha I$. For $S \in K_{\alpha, \beta}$, define

$$
F(S):=\sum_{i=1}^{p} \lambda_{i}\left(S^{1 / 2} S_{i} S^{1 / 2}\right)^{1 / 2}
$$

It is easy to see that

$$
\beta I \geq \sum_{i=1}^{p} \lambda_{i} \sqrt{\beta \beta_{i}} I \geq F(S) \geq \sum_{i=1}^{p} \lambda_{i} \sqrt{\alpha \alpha_{i}} I \geq \alpha I, \quad \forall S \in K_{\alpha, \beta} .
$$

Then $F$ is a self-map of $K_{\alpha, \beta}$. It is also continuous on $K_{\alpha, \beta}$. The existence of a solution to (6.2) in $K_{\alpha, \beta}$ then directly follows from Brouwer's fixed-point theorem.

Step 2: sufficiency. Set $\bar{\nu}:=\mathcal{N}(0, \bar{S})$ where $\bar{S}$ is a positive definite solution of (6.2). The optimal transport between $\bar{\nu}$ and $\nu_{i}$ is then the linear map

$$
T_{i}=S_{i}^{1 / 2}\left(S_{i}^{1 / 2} \bar{S} S_{i}^{1 / 2}\right)^{-1 / 2} S_{i}^{1 / 2}
$$


Let us now prove that $\sum_{i=1}^{p} \lambda_{i} T_{i}=I$ that we already know, from proposition 3.8 , to be a sufficient condition for $\bar{\nu}$ to solve (2.2). Set $K_{i}=S_{i}^{1 / 2}$ and $\bar{K}:=\bar{S}^{1 / 2}$. Using the identity

$$
\left(\bar{K} K_{i}^{2} \bar{K}\right)^{1 / 2}=\bar{K} K_{i}\left(K_{i} \bar{K}^{2} K_{i}\right)^{-1 / 2} K_{i} \bar{K}
$$

we may rewrite $F(\bar{S})=\bar{S}$ as

$$
\sum_{i=1}^{p} \lambda_{i} \bar{K} K_{i}\left(K_{i} \bar{K}^{2} K_{i}\right)^{-1 / 2} K_{i} \bar{K}=\bar{K}^{2}
$$

and since $\bar{K}$ is invertible, this yields

$$
\sum_{i=1}^{p} \lambda_{i} K_{i}\left(K_{i} \bar{K}^{2} K_{i}\right)^{-1 / 2} K_{i}=\sum_{i=1}^{p} \lambda_{i} T_{i}=I
$$

which proves that $\bar{\nu}$ is optimal.

Step 3: We already know that (2.2) admits a unique solution and from the previous step, we have for any positive definite solution $\bar{S}$ of $(6.2), \mathcal{N}(0, \bar{S})$ solves (2.2). This proves that (6.2) has a unique positive definite solution.

The crucial role played by the nonlinear matrix equation (6.2) and the sufficiency step in the proof above is due to Knott and Smith [9]. But the authors left open the existence and uniqueness issues for (6.2); indeed the fact that barycenters of gaussians are also gaussians was not proved in their paper. Let us point out here that Rüschendorf and Uckelman proved in [13] existence of a solution to (6.2) by a completely different argument than Brouwer's fixed point, but they did not study its uniqueness.

\section{Convex functionals}

Here we extend the notion of displacement convexity introduced by McCann [11] for two probability measures, to any finite number of probability measures. For simplicity, we assume from now on that all probability measures are absolutely continuous with respect to the Lebesgue measure. Also, the space $X^{\prime} \cap \mathcal{M}_{+}^{1}\left(\mathbb{R}^{d}\right)$ will be equipped with the Wasserstein metric (2.1); it will then be called the Wasserstein space.

Definition 7.1. A functional $\mathcal{F}: X^{\prime} \cap \mathcal{M}_{+}^{1}\left(\mathbb{R}^{d}\right) \rightarrow \mathbb{R}$ is said to be convex along barycenters in the Wasserstein space $X^{\prime} \cap \mathcal{M}_{+}^{1}\left(\mathbb{R}^{d}\right)$, if given any $p \geq$ 
2 probability measures $\nu_{1}, \cdots, \nu_{p}$ in $X^{\prime} \cap \mathcal{M}_{+}^{1}\left(\mathbb{R}^{d}\right)$ and any $p$ positive real numbers $\lambda_{1}, \cdots, \lambda_{p}$ that sum to 1 , we have

$$
\mathcal{F}\left(\operatorname{bar}\left(\nu_{i}, \lambda_{i}\right)_{i=1, \cdots, p}\right) \leq \sum_{i=1}^{p} \lambda_{i} \mathcal{F}\left(\nu_{i}\right),
$$

where $\operatorname{bar}\left(\nu_{i}, \lambda_{i}\right)_{i=1, \cdots, p}$ denotes the barycenter of the probability measures $\nu_{i}$ with the weights $\lambda_{i}$.

To compare the convexity along barycenters with McCann's displacement convexity, let us first recall the definition of displacement convexity as introduced in [11].

Definition 7.2. A functional $\mathcal{F}: X^{\prime} \cap \mathcal{M}_{+}^{1}\left(\mathbb{R}^{d}\right) \rightarrow \mathbb{R}$ is said to be displacement convex in the Wasserstein space $X^{\prime} \cap \mathcal{M}_{+}^{1}\left(\mathbb{R}^{d}\right)$, if given any two probability measures $\nu_{0}$ and $\nu_{1}$ in $X^{\prime} \cap \mathcal{M}_{+}^{1}\left(\mathbb{R}^{d}\right)$, the function $[0,1] \ni t \mapsto$ $\mathcal{F}\left(\nu_{t}\right) \in \mathbb{R}$ is convex; here $\nu_{t}$ is McCann's interpolant between $\nu_{0}$ and $\nu_{1}$ given by $\nu_{t}=((1-t) \mathrm{id}+t T) \sharp \nu_{0}$, where $T=\nabla \phi$ is Brenier's map transporting $\nu_{0}$ to $\nu_{1}$.

The following proposition shows that the convexity along barycenters generalizes McCann's displacement convexity.

Proposition 7.3. If a functional $\mathcal{F}: X^{\prime} \cap \mathcal{M}_{+}^{1}\left(\mathbb{R}^{d}\right) \rightarrow \mathbb{R}$ is convex along barycenters, then it is displacement convex.

Proof. Let $\nu_{0}, \nu_{1} \in X^{\prime} \cap \mathcal{M}_{+}^{1}\left(\mathbb{R}^{d}\right)$ and $t \in[0,1]$. From section 6.2 , we have that the barycenter of $\nu_{0}$ and $\nu_{1}$ with weights $\lambda_{0}=1-t$ and $\lambda_{1}=t$ respectively, is McCann's interpolant $\nu_{t}$. Then if $\mathcal{F}$ is convex along barycenters, then (7.1) implies that

$$
\mathcal{F}\left(\nu_{t}\right) \leq(1-t) \mathcal{F}\left(\nu_{0}\right)+t \mathcal{F}\left(\nu_{1}\right),
$$

which proves the displacement convexity of $\mathcal{F}$.

Contrarily to the Euclidean case, definitions 7.1 and 7.2 may not be equivalent, except in dimension $d=1$ (see proposition below). But they are related to a third notion of convexity, that is the convexity along generalized geodesics joining any two probability measures $\nu_{1}$ and $\nu_{2}$ with base a probability measure $\nu$ (see [1]). In fact, the convexity along generalized geodesics is the strongest among these three notions of convexity, while McCann's displacement convexity is the weakest. Before justifying this statement, let us first recall the definition of generalized geodesics and its corresponding convexity, as presented in [1]. 
Definition 7.4. Let $\nu_{1}, \nu_{2}, \nu \in X^{\prime} \cap \mathcal{M}_{+}^{1}\left(\mathbb{R}^{d}\right)$. Denote by $T_{i}=\nabla \phi_{i}$ Brenier's map transporting $\nu$ to $\nu_{i}$ for $i=1,2$. Then the generalized geodesic joining $\nu_{1}$ and $\nu_{2}$ with base $\nu$ is the interpolated curve

$$
\left[\nu_{1}, \nu_{2}\right]_{t}^{\nu}:=\left((1-t) T_{1}+t T_{2}\right) \sharp \nu, \quad t \in[0,1] .
$$

Moreover, a functional $\mathcal{F}: X^{\prime} \cap \mathcal{M}_{+}^{1}\left(\mathbb{R}^{d}\right) \rightarrow \mathbb{R}$ is said to be convex along generalized geodesics, if given any three probability measures $\nu_{1}, \nu_{2}, \nu \in X^{\prime} \cap$ $\mathcal{M}_{+}^{1}\left(\mathbb{R}^{d}\right)$, we have

$$
\mathcal{F}\left(\left[\nu_{1}, \nu_{2}\right]_{t}^{\nu}\right) \leq(1-t) \mathcal{F}\left(\nu_{1}\right)+t \mathcal{F}\left(\nu_{2}\right),
$$

where $\left[\nu_{1}, \nu_{2}\right]_{t}^{\nu}$ is defined as above.

The definition of convexity along generalized geodesics could as well be given with any finite number of probability measures. Indeed, we have:

Proposition 7.5. A functional $\mathcal{F}: X^{\prime} \cap \mathcal{M}_{+}^{1}\left(\mathbb{R}^{d}\right) \rightarrow \mathbb{R}$ is convex along generalized geodesics if and only if, given any $p \geq 2$ probability measures $\nu_{1}, \cdots, \nu_{p} \in X^{\prime} \cap \mathcal{M}_{+}^{1}\left(\mathbb{R}^{d}\right)$, any $p$ positive real numbers $\lambda_{1}, \cdots, \lambda_{p}$ that sum to 1 , and a reference measure $\nu \in X^{\prime} \cap \mathcal{M}_{+}^{1}\left(\mathbb{R}^{d}\right)$, we have

$$
\mathcal{F}\left(\left[\nu_{1}, \cdots, \nu_{p}\right]_{\lambda_{i}}^{\nu}\right) \leq \sum_{i=1}^{p} \lambda_{i} \mathcal{F}\left(\nu_{i}\right)
$$

where

$$
\left[\nu_{1}, \cdots, \nu_{p}\right]_{\lambda_{i}}^{\nu}:=\left(\sum_{i=1}^{p} \lambda_{i} \nabla \Phi_{i}\right) \sharp \nu
$$

and $\nabla \Phi_{i}$ is Brenier's map transporting $\nu$ to $\nu_{i}$.

Proof. The proof is done by induction on the number $p$ of probability measures. We start with three measures $\nu_{1}, \nu_{2}, \nu_{3}$ in $X^{\prime} \cap \mathcal{M}_{+}^{1}\left(\mathbb{R}^{d}\right)$ with respective weights $\lambda_{1}, \lambda_{2}, \lambda_{3}$ such that $\sum_{i=1}^{3} \lambda_{i}=1$. We have that $\sum_{i=1}^{3} \lambda_{i} \nabla \Phi_{i}=$ $\lambda_{12} \nabla \Phi_{12}+\lambda_{3} \nabla \Phi_{3}$, where $\lambda_{12}=\lambda_{1}+\lambda_{2}$, and $\Phi_{12}=\frac{\lambda_{1}}{\lambda_{12}} \Phi_{1}+\frac{\lambda_{2}}{\lambda_{12}} \Phi_{2}$ is convex. Since $\lambda_{12}+\lambda_{3}=1=\left(\lambda_{1} / \lambda_{12}\right)+\left(\lambda_{2} / \lambda_{12}\right)$, then using (7.5) and (7.3), we have

$$
\begin{aligned}
\mathcal{F}\left(\left[\nu_{1}, \nu_{2}, \nu_{3}\right]_{\lambda_{i}}^{\nu}\right) & =\mathcal{F}\left(\left(\lambda_{12} \nabla \Phi_{12}+\lambda_{3} \nabla \Phi_{3}\right) \sharp \nu\right) \\
& \leq \lambda_{12} \mathcal{F}\left(\nabla \Phi_{12} \sharp \nu\right)+\lambda_{3} \mathcal{F}\left(\nabla \Phi_{3} \sharp \nu\right) \\
& \leq \lambda_{12}\left(\frac{\lambda_{1}}{\lambda_{12}} \mathcal{F}\left(\nabla \Phi_{1} \sharp \nu\right)+\frac{\lambda_{2}}{\lambda_{12}} \mathcal{F}\left(\nabla \Phi_{2} \sharp \nu\right)\right)+\lambda_{3} \mathcal{F}\left(\nabla \Phi_{3} \sharp \nu\right) \\
& =\sum_{i=1}^{3} \lambda_{i} \mathcal{F}\left(\nabla \Phi_{i} \sharp \nu\right)=\sum_{i=1}^{3} \lambda_{i} \mathcal{F}\left(\nu_{i}\right) .
\end{aligned}
$$

(7.4) then readily follows by an induction argument on $p$. 
The next proposition shows that convexity along barycenters is intermediate to the other two notions of convexity.

Proposition 7.6. If $\mathcal{F}: X^{\prime} \cap \mathcal{M}_{+}^{1}\left(\mathbb{R}^{d}\right) \rightarrow \mathbb{R}$ is convex along generalized geodesics, then it is convex along barycenters (and therefore displacement convex because of proposition 7.3).

Moreover if $d=1$, then displacement convexity implies convexity along generalized geodesics; hence in this case, these three notions of convexity are equivalent.

Proof. Let $\nu_{1}, \cdots, \nu_{p} \in X^{\prime} \cap \mathcal{M}_{+}^{1}\left(\mathbb{R}^{d}\right)$, and consider the barycenter $\operatorname{bar}\left(\nu_{i}, \lambda_{i}\right)_{i=1, \cdots, p}$ of these measures with respective weights $\lambda_{1}, \cdots, \lambda_{p}$. From the multi-marginal characterization (4.10) of barycenters, we have

$$
\operatorname{bar}\left(\nu_{i}, \lambda_{i}\right)_{i=1, \cdots, p}=\left(\sum_{i=1}^{p} \lambda_{i} \nabla u_{i}^{*} \circ \nabla u_{1}\right) \sharp \nu_{1}=\left(\sum_{i=1}^{p} \lambda_{i} \nabla u_{i}^{*}\right) \sharp \tilde{\nu}_{1}
$$

where $\tilde{\nu}_{1}:=\nabla u_{1} \sharp \nu_{1}$, the $u_{i}$ are strictly convex on $\mathbb{R}^{d}$, and the $T_{i}^{1}=\nabla u_{i}^{*} \circ \nabla u_{1}$ satisfy $T_{i}^{1} \sharp \nu_{1}=\nu_{i}$. So if $\mathcal{F}$ is convex along generalized geodesics, then using (7.4) and (7.5), we conclude that

$$
\mathcal{F}\left(\left(\operatorname{bar}\left(\nu_{i}, \lambda_{i}\right)_{i=1, \cdots, p}\right) \leq \sum_{i=1}^{p} \lambda_{i} \mathcal{F}\left(\nabla u_{i}^{*} \sharp \tilde{\nu}_{1}\right)=\sum_{i=1}^{p} \lambda_{i} \mathcal{F}\left(T_{i}^{1} \sharp \nu_{1}\right)=\sum_{i=1}^{p} \lambda_{i} \mathcal{F}\left(\nu_{i}\right),\right.
$$

that is the convexity of $\mathcal{F}$ along barycenters.

Next assume that $d=1$, and consider $\nu, \nu_{0}, \nu_{1} \in X^{\prime} \cap \mathcal{M}_{+}^{1}(\mathbb{R})$. Then Brenier's map transporting $\nu$ to $\nu_{i}$ is $\Phi_{i}^{\prime}$, where $\Phi_{i}$ is nondecreasing for $i=$ 0 , 1. So $T=\Phi_{1}^{\prime} \circ\left(\Phi_{0}^{\prime}\right)^{-1}$ is nondecreasing and $T \sharp \nu_{0}=\nu_{1}$, so that $T$ is Brenier's map transporting $\nu_{0}$ to $\nu_{1}$. Therefore, the generalized geodesic joining $\nu_{0}$ and $\nu_{1}$ with base $\nu$ coincides with McCann's geodesic, since

$$
\left[\nu_{0}, \nu_{1}\right]_{t}^{\nu}=\left[(1-t) \Phi_{0}^{\prime}+t \Phi_{1}^{\prime}\right] \sharp\left(\left(\Phi_{0}^{\prime}\right)^{-1} \sharp \nu_{0}\right)=[(1-t) \mathrm{id}+t T] \sharp \nu_{0} .
$$

Hence if $\mathcal{F}$ is displacement convex, we have

$$
\begin{aligned}
\mathcal{F}\left(\left[\nu_{0}, \nu_{1}\right]_{t}^{\nu}\right) & =\mathcal{F}\left([(1-t) \mathrm{id}+t T] \sharp \nu_{0}\right) \\
& \leq(1-t) \mathcal{F}\left(\nu_{0}\right)+t \mathcal{F}\left(T \sharp \nu_{0}\right) \\
& =(1-t) \mathcal{F}\left(\Phi_{0}^{\prime} \sharp \nu\right)+t \mathcal{F}\left(\Phi_{1}^{\prime} \sharp \nu\right),
\end{aligned}
$$

which shows that $\mathcal{F}$ is convex along generalized geodesics. 
We end this section by remarking that the three basic examples of functionals, i.e. the internal energy, the potential energy and the interaction energy, are convex along barycenters, since they are all convex along generalized geodesics in $X^{\prime} \cap \mathcal{M}_{+}^{1}\left(\mathbb{R}^{d}\right)$ as shown by Ambrosio, Gigli and Savaré in [1], propositions 9.3.2, 9.3.5 and 9.3.9. In the next statement, we identify the absolutely continuous probability measures belonging to $X^{\prime}$ with their density functions that we denote by $\rho$.

Proposition 7.7. Let $F:[0, \infty) \rightarrow \mathbb{R}$ be continuous on $[0, \infty)$, and $V, W$ : $\mathbb{R}^{d} \rightarrow \mathbb{R}$ be continuous on $\mathbb{R}^{d}$, with $W$ being an even function.

1. If $F(0)=0$ and $(0, \infty) \ni t \mapsto t^{d} F\left(t^{-d}\right)$ is convex and nonincreasing, then the internal energy $E(\rho)=\int_{\mathbb{R}^{d}} F(\rho(x)) d x$ is convex along generalized geodesics in $X^{\prime} \cap \mathcal{M}_{+}^{1}\left(\mathbb{R}^{d}\right)$.

2. If $V$ is convex, then the potential energy $E(\rho)=\int_{\mathbb{R}^{d}} V(x) \rho(x) d x$ is convex along generalized geodesics in $X^{\prime} \cap \mathcal{M}_{+}^{1}\left(\mathbb{R}^{d}\right)$.

3. If $W$ is convex, then the interaction energy

$$
E(\rho)=\frac{1}{2} \int_{\mathbb{R}^{d}}(W \star \rho)(x) \rho(x) d x=\frac{1}{2} \iint_{\mathbb{R}^{d} \times \mathbb{R}^{d}} W(x-y) \rho(x) \rho(y) d x d y
$$

is convex along generalized geodesics in $X^{\prime} \cap \mathcal{M}_{+}^{1}\left(\mathbb{R}^{d}\right)$.

Therefore, all these functionals are convex along barycenters in the Wasserstein space $X^{\prime} \cap \mathcal{M}_{+}^{1}\left(\mathbb{R}^{d}\right)$.

Acknowledgments This work was completed during a stay of the first author at the CEREMADE, for which the authors gratefully acknowledge the support of the Agence Nationale de la Recherche (ANR) through the project ANR-09-JCJC-0096-01 EVAMEF. G.C. also wishes to thank the support of the ANR through the project ANR-07-BLAN-0235 OTARIE. M.A acknowledges the support of the Natural Sciences and Engineering Research Council of Canada for a Discovery grant.

\section{References}

[1] L. Ambrosio, N. Gigli, and G. Savaré, gradient flows in metric spaces and in the space of probability measures, Lectures in Mathematics, Birkhäuser (2005).

[2] M. Bernot, J. Delon, G. Peyré, J. Rabin, Wasserstein Barycenter and its Application to Texture Mixing, preprint 2010. 
[3] Y. Brenier, Polar factorization and monotone rearrangement of vectorvalued functions, Comm. Pure Appl. Math. 44 4, pp. 375-417 (1991).

[4] G. Carlier, I. Ekeland, Matching for teams, Econom. Theory, 42, no. 2, pp. 397-418 (2010).

[5] P.-A. Chiappori, R.J. McCann, L. Nesheim, Hedonic price equilibria, stable matching, and optimal transport: equivalence, topology, and uniqueness, Econom. Theory, 42, no. 2, pp.317-354 (2010).

[6] C. Dellacherie, P.-A. Meyer, Probabilities and Potential, Mathematical Studies 29, North-Holland, 1978.

[7] I. Ekeland, R. Temam, Convex Analysis and Variational Problems, Classics in Mathematics, Society for Industrial and Applied Mathematics, Philadelphia, (1999).

[8] W. Gangbo, A. Święch, Optimal maps for the multidimensional MongeKantorovich problem, Comm. Pure Appl. Math., 51, no. 1, pp. 23-45 (1998).

[9] M. Knott and C. S. Smith, On a generalization of cyclic-monotonicity and distances among random vectors, Linear Algebra and its Appl., 199, pp. 363-371 (1994).

[10] R.J. McCann, Existence and uniqueness of monotone measurepreserving maps, Duke Math. J., 80, pp. 309-323, (1995)

[11] R.J. McCann, A convexity principle for interacting gases, Advances in Mathematics, 128, pp. 153-179 (1997).

[12] S. Ohta, Barycenters in Alexandrov spaces of curvature bounded below, preprint 2010.

[13] L. Rüschendorf, L. Uckelmann, On the n-coupling problem, Journal of Multivariate Analysis 81, pp. 242-258 (2002).

[14] K.T. Sturm, Probability measures on metric spaces of nonpositive curvature, Contemp. Math. 338, pp.357-390 (2003).

[15] C. Villani, Topics in optimal transportation, Graduate Studies in Mathematics, 58, American Mathematical Society, Providence, RI, 2003. 